\title{
Immunity, neuroglia and neuroinflammation in autism
}

\author{
CARLOS A. PARDO ${ }^{1,2,3}$, DIANA L. VARGAS ${ }^{1,2}$, \& ANDREW W. ZIMMERMAN ${ }^{1,4}$ \\ ${ }^{1}$ Department of Neurology, ${ }^{2}$ Division of Neuroimmunology and Infectious Disorders, ${ }^{3}$ Department of Pathology, \\ Fohns Hopkins University School of Medicine, Baltimore, and ${ }^{4}$ Kennedy Krieger Institute, Baltimore, Maryland, USA
}

\begin{abstract}
Summary
Autism is a complex neurodevelopmental disorder of early onset that is highly variable in its clinical presentation. Although the causes of autism in most patients remain unknown, several lines of research support the view that both genetic and environmental factors influence the development of abnormal cortical circuitry that underlies autistic cognitive processes and behaviors. The role of the immune system in the development of autism is controversial. Several studies showing peripheral immune abnormalities support immune hypotheses, however until recently there have been no immune findings in the CNS. We recently demonstrated the presence of neuroglial and innate neuroimmune system activation in brain tissue and cerebrospinal fluid of patients with autism, findings that support the view that neuroimmune abnormalities occur in the brain of autistic patients and may contribute to the diversity of the autistic phenotypes. The role of neuroglial activation and neuroinflammation are still uncertain but could be critical in maintaining, if not also in initiating, some of the CNS abnormalities present in autism. A better understanding of the role of neuroinflammation in the pathogenesis of autism may have important clinical and therapeutic implications.
\end{abstract}

\section{Introduction}

Autism is the most severe and devastating condition in the broad spectrum of developmental disorders called 'pervasive developmental disorders' (Rapin, 1997). Autistic disorders are characterized by marked impairment in social skills, verbal communication, behavior, and cognitive function (Rapin, 1997; Lord et al., 2000). Abnormalities in language development, mental retardation, and epilepsy are frequent problems in the clinical profile of patients with autism (Rapin, 1997). The syndrome is clinically heterogeneous and can be associated in up to $10 \%$ of patients with well-described neurological and genetic disorders, such as tuberous sclerosis, fragile $\mathrm{X}$, Rett and Down syndromes, although in most patients the causes are still unknown (Rapin \& Katzman, 1998; Newschaffer et al., 2002; Cohen et al., 2005). The importance of autism as a public health problem has been recognized in recent years, as epidemiological studies have suggested that the age-adjusted incidence of research-identified autism has increased from 5.5 (95\% confidence interval, 1.4-9.5) per 100000 children in the period $1980-1983$ to 44.9 (95\% confidence interval, 32.9-56.9) in the period 1995-1997 (8.2-fold increase) (Barbaresi et al., 2005) while the prevalence of autistic syndromes has increased to 3-6 per 1000 children, with a male to female ratio of 3:1 (Fombonne, 2003; YearginAllsopp et al., 2003). In addition to complex multigenic factors (Folstein \& Rosen-Sheidley, 2001), several researchers have also hypothesized important roles for environmental factors, pre- or perinatal injuries, vaccines, mercury toxicity, or persistent viral infections (Wing \& Potter, 2002; Larsson et al., 2005). These recent observations on the epidemiology of autism strongly suggest that an interplay between genetic and as yet undefined environmental factors may increase the risk of autism, to a level greater than one would expect from genetic causes alone.

\section{Neurobiology of autism \\ Clinical and epidemiological aspects of autism}

Although the neurobiological basis for autism remains poorly understood, several lines of research now support the view that genetic, environmental, neurological, and immunological factors contribute to its development (Rapin \& Katzman, 1998; Newschaffer et al., 2002; Folstein \& RosenSheidley, 2001; Korvatska et al., 2002). Several different genetic factors and/or other risk factors may combine during development to produce complex

Correspondence: Carlos A. Pardo, MD, Department of Neurology, Johns Hopkins University School of Medicine, Pathology 627, 600 North Wolfe Street, Baltimore, Maryland 21287, USA. Tel: +410 614 5757. Fax: +410 502 7609. E-mail: cpardo@jhmi.edu 
changes in CNS organization that translate into abnormalities of neuronal and cortical cytoarchitecture that are responsible for the complex language and behavioral problems that characterize the autistic phenotype. The core symptoms of autism include abnormal communication, social relatedness, behavior, and cognition (Rapin, 1997; Lord et al., 2000). The majority of children show abnormalities during infant development that may not become apparent until the second year of life. Approximately $30-50 \%$ of children undergo regression, with a loss of skills, including language, between 16 and 25 months of age (Lord et al., 2004). In the medical evaluation of autism, specific etiologies can be found in $<10 \%$ of children, including fragile $\mathrm{X}$, tuberous sclerosis, and other rare diseases (Cohen et al., 2005). Epilepsy occurs in up to $40 \%$ of patients, and epileptic discharges may occur on EEGs early in childhood, even in the absence of clinical seizures (Tuchman \& Rapin, 2002). Although children with autism present with a wide spectrum of symptoms that vary in severity and clinical progression, it is possible to define these features in affected individuals and follow them over time (Aman et al., 2004).

\section{Neuroanatomical abnormalities in autism}

A wide range of anatomical and structural brain abnormalities have been observed in autistic patients by longitudinal clinical and magnetic resonance imaging studies. The most remarkable observation is that the clinical onset of autism appears to be preceded by two phases of brain growth abnormalities: a reduced head size at birth and a sudden and excessive increase in head size between 1-2 months and 6-14 months (Courchesne et al., 2004). These studies have also shown that the most abnormal pattern of brain overgrowth occurs in areas of the frontal lobe, cerebellum, and limbic structures between 2-4 years of age, a pattern that is followed by abnormal slowness and an arrest in brain growth (Courchesne et al., 2004; Courchesne \& Pierce, 2005). Other studies of high-functioning autistic patients have shown an overall enlargement of brain volume associated with increased cerebral white matter and decrease in cerebral cortex and hippocampal-amygdala volumes (Herbert et al., 2003; Herbert et al., 2004). One of the most puzzling issues in the neuroanatomical observations in autism is the lack of an acceptable explanation for the cause of this dissociation or patterns of abnormal brain growth. However, it is likely that disruption of white matter tracts and disconnection between brain regions are present in autistic patients, as demonstrated by new techniques such as diffusion tensor imaging. This approach has demonstrated reduced fractional anisotropy values in white matter adjacent to the ventromedial prefrontal cortices, anterior cingulate gyrus, and superior temporal regions, findings suggestive of the disruption in white matter tracts in brain regions involved in social functioning that has been described in autistic patients (Barnea-Goraly et al., 2004).

In addition to abnormal growth patterns of the brain, one of the most consistent findings of neuroimaging studies in autism is the presence of abnormalities in the cerebellum. Reduction in the size of cerebellar regions such as the vermis (Hashimoto et al., 1995; Kaufmann et al., 2003), an increase in white matter volume, and reduction in the gray/white matter ratio (Courchesne \& Pierce, 2005) are the most prominent changes observed in the cerebellum. In one of these studies, the cerebellar changes appeared to be specific to autism, in contrast to other neurodevelopmental disorders such as Down syndrome, Down syndrome with autism, fragile $\mathrm{X}$ and fragile $\mathrm{X}$ with autism (Kaufmann et al., 2003). These observations concur with: (1) the findings from neuropathological studies describing abnormalities in the cerebellum, such as a decreased number of Purkinje cells (Kemper \& Bauman, 1998; Bailey et al., 1998) and, most recently, (2) observation of increased microglial activation and astroglial reactions in both the granular cell and white matter layers and a reduction in Purkinje and granular cells (Vargas et al., 2005).

\section{Neuropathology of autism}

Cytoarchitectural organizational abnormalities of the cerebral cortex, cerebellum, and other subcortical structures appear to be the most prominent neuropathological changes in autism (Kemper \& Bauman, 1998; Bailey et al., 1998). An unusual laminar cytoarchitecture with packed small neurons has been described in the classical neuropathological studies by Kemper and Bauman (1998), but no abnormalities in the external configuration of the cerebral cortex were noted. Cerebellar and brainstem pathology was prominent, with a loss and atrophy of Purkinje cells, predominantly in the posterolateral neocerebellar cortex. Kemper and Bauman (1998) have delineated at least three different types of pathological abnormalities in autism: (1) a curtailment of the normal development of neurons in the forebrain limbic system; (2) an apparent decrease in the cerebellar Purkinje cell population; and (3) agerelated changes in neuronal size and number in the nucleus of the diagonal band of Broca, the cerebellar nuclei, and the inferior olive. These observations suggest that delays in neuronal maturation are important component in the spectrum of 
neuropathological changes in autism (Kemper \& Bauman, 1998). In addition to these cytoarchitectural abnormalities, the number of cortical minicolumns, the narrow chain of neurons that extend vertically across layers $2-6$ to form anatomical and functional units, appeared to be more numerous, smaller, and less compact in their cellular configuration in the frontal and temporal regions of the brain of autistic patients, as compared with controls (Casanova et al., 2002). Pathological evidence of immunological reactions within the CNS, such as lymphocyte infiltration and microglial nodules, has been described in a few case reports (Bailey et al., 1998; Guerin et al., 1996).

\section{Immunological factors associated with autism}

\section{Immunological abnormalities in autism}

Reports of differences in systemic immune findings over the past 30 years have led to speculation that autism may represent, in some patients, an immune mediated or autoimmune disorder (Ashwood \& de Water, 2004). Recent reviews of immune dysfunction in autism have sought to understand these findings in the clinical context of the syndrome (Korvatska et al., 2002; Ashwood \& de Water, 2004; Zimmerman, 2005). Abnormalities of both humoral and cellular immune functions have been described in small studies of children with autism $(N=10-36)$, and include decreased production of immunoglobulins or B and T-cell dysfunction (Warren et al., 1986). Early studies suggested that prenatal viral infections might damage the immature immune system and induce viral tolerance (Stubbs \& Crawford, 1977), while later studies showed altered T-cell subsets and activation, consistent with the possibility of an autoimmune pathogenesis (Gupta et al., 1998). Odell et al. (2005) recently confirmed earlier reports of a four-fold increase in the serum complement (C4B) null allele (i.e., no protein produced) in 85 children with autism, compared to controls.

Studies of peripheral blood have shown a range of abnormalities, including T-cell, B-cell, and NK-cell dysfunction; autoantibody production; and increased pro-inflammatory cytokines (Gupta et al., 1998; Singh et al., 1997; Singh et al., 2002; Vojdani et al., 2002; Jyonouchi et al., 2001). Shifts observed in Th1 to Th2 lymphocyte subsets and cytokines and associations with human leukocyte antigen (HLA)DR4 have suggested the possibility that autoimmunity against brain antigens may contribute to the neuropathology of autism (van Gent et al., 1997). Decreases in immunoglobulin subsets and complement, the presence of auto-antibodies against CNS antigens, and an effect of maternal antibodies have also been proposed as pathogenic factors (Dalton et al., 2003). In most of these studies, phenotyping was limited to descriptions of the subjects as 'autistic' based on criteria of the Diagnostic and Statistical Manual of the American Psychiatric Association. 'Abnormal' immune findings varied from $15-60 \%$ of children with autism. For some parameters, unaffected siblings showed intermediate values, and a background of such 'abnormalities' was noted in normal controls as well. In all studies, measurements have been reported at single time points and among subjects of different ages. Since these differences in systemic immune findings in autism have not been followed in the same patients over time, it is not clear whether they reflect true immune dysfunction or may represent dysmaturation that changes with age (Zimmermann, 2005). Also, no clinical immune deficiency states have been reported in association with unusual infections or reactions to immunizations, despite widespread interest in the possibility of such relationships (Halsey \& Hyman, 2001).

\section{Autoimmunity and autism}

Circulating auto-antibodies directed against CNS antigens have been described in patients with autism, reacting to myelin basic protein (Singh, Lin, \& Tang, 1998), frontal cortex (Todd et al., 1988), cerebral endothelial cells (Connolly et al., 1999), and neurofilament proteins (Singh et al., 1997). Autoreactivity to a human protein with molecular weight in the range (but distinct from) myelin basic protein has been reported (Silva et al., 2004). Recent findings suggest reactivity in sera from children with autism to a $73 \mathrm{Kd}$ epitope in the cingulate gyrus and cerebellum. The significance of auto-antibodies in serum from patients with autism has been difficult to determine. Their presence might imply that autism is an autoimmune disorder. However, several criteria, including the necessity to demonstrate the autoimmune disease after passive transfer of antibodies into animals, would be necessary to establish the role of these auto-antibodies as pathogenic effectors (Rose \& Bona, 1993), and this evidence is still lacking. Even though several antibodies in autism serum have been demonstrated to react against human brain tissue, their pathogenicity has not been demonstrated in autism postmortem brain tissue. Of equal interest to serum reactivity in the children, however, have been studies in maternal sera. Warren et al. (1990) demonstrated reactivity of mothers' sera to their autistic children's lymphocytes. Maternal serum has also been shown to cause antibody binding to fetal Purkinje cells when it was injected into pregnant mice (Dalton et al., 2003). Maternal antibodies may therefore be relevant 
to prenatal brain development (Dalton et al., 2003), by interfering with cell signaling in the developing brain, and (perhaps) disturbing patterns of CNS organization.

Other autoimmune disorders, such as rheumatoid arthritis, lupus and thyroid disorders, have been found at increased rates in surveys of family members of children with autism, rather than in the children themselves, compared to controls. This was first observed in one family by Money (Money, Bobrow, \& Clarke, 1971), and subsequently in three clinical surveys (Comi et al., 1999; Sweeten et al., 2003; Molloy CA, personal communication). However, these associations were not found in another study after review of medical records (Micali, Chakrabarti, \& Fombonne, 2004). A recent study of mothers with autistic children reported an association with psoriasis but not other autoimmune disorders, and a two-fold increased risk of having an autistic child for those mothers with asthma and allergies during the second trimester (Croen et al., 2005). The meaning of these studies for autism is still not clear, but they suggest that maternal immunological effects might be important during gestation. They are also consistent with reported increases in frequencies of HLA DR4 and related alleles in children with autism and their mothers (Daniels et al., 1995; Torres et al., 2002).

\section{Immunogenetics in autism}

Some of the most promising studies that link the immune system to autism come from the study of the HLA genes, which are important genetic determinants of immune function within the major histocompatibility complex (MHC) and could reflect important antigenic differences between parents and their affected children. Other genetic loci associated with autoimmune and inflammatory disorders appear to cluster with those for autism (as well as Tourette's syndrome) and suggest a genetic relationship based on immune dysregulation (Becker, Freidlin, \& Simon, 2003). In the case of HLA genes, the association of specific antigens/ alleles with autoimmunity suggests that autistic patients may exhibit a similar pattern of association. Immunogenetic studies have shown an increased frequency of HLA-DR4 in children with autism and their mothers, a finding that is consistent with clinical observations of increased frequencies of autoimmune disorders in families with autism (although not in the children themselves) (Comi et al., 1999). These observations are important, as HLA-DR4, a class II antigen, has been identified as one of the susceptibility markers for certain autoimmune diseases, such as rheumatoid arthritis, and is strongly associated with others such as hypothyroidism and autoimmune diabetes (Levin et al., 2004). These disorders have a higher incidence among families, especially mothers, of autistic children than of controls (Comi et al., 1999; Sweeten et al., 2003). These findings were further supported by a recent report that DR4 alleles occur in individuals with autism with higher frequency than in controls recruited from the National Marrow Donor Program (Torres et al., 2002). These observations have led researchers to investigate the possible expression of HLA-DR4 in the families of some children with autism. To confirm this possible association between HLA-DR4 and autism, we studied HLA-DR4 and its subtypes in single-birth and multiplex families with autism (Zimmerman, Tyler, \& Matteson, 2001). Among 17 single-birth families with an autistic child in the East Tennessee region, the mothers were 4.62 times more likely (95\% CI: $1.54,14.34)$, and the children were 3.6 times more likely to have an HLA-DR4 haplotype than were controls (Lee et al., 2004).

\section{Infections and autism}

Infections have been associated with autism in small numbers of children, and include prenatal rubella (Chess, Fernandez, \& Korn, 1978) and cytomegalovirus (Sweeten et al., 2003; Yamashita et al., 2003), and postnatal herpes encephalitis (DeLong, Bean, \& Brown, 1981). Given the variety of viruses and their pathogenic effects that can be associated with autism, the location of the pathology and the neural networks affected appear to be more important than the specific types of viruses. For example, reversible symptoms of autism have been reported with bilateral temporal lobe involvement in herpes simplex virus encephalitis (DeLong, Bean, \& Brown, 1981). Autism rarely results from known infectious causes, and the immune abnormalities or variants described in autism studies have not been consistent with typical immune deficiency states that would predispose to such infections. Furthermore, there have been no documented increased rates of infection in children with autism (Comi et al., 1999). And, although persistence of measles virus in the GI tract and peripheral mononuclear cells has been reported in children with autism (Kawashima et al., 2000), replication and further study of its possible relevance to autism in CSF and brain tissue are needed. Animal models of autism using prenatal infections (Patterson, 2002) lend credence to the importance of gestational effects on fetal brain development, as in the association of maternal influenza and the increased risk of schizophrenia (Shi et al., 2003). Autistic behaviors also have been 
induced experimentally in a rat model using neonatal Borna disease virus (Carbone et al., 2002).

\section{Neuroglia responses and neuroinflammation in autism}

\section{Neuroglia and CNS function}

Neuroglial cells such as astrocytes and microglia, along with perivascular macrophages and endothelial cells, play important roles in neuronal function and homeostasis (Aloisi, 2001; Dong \& Benveniste, 2001). Both microglia and astroglia are fundamentally involved in cortical organization, neuroaxonal guidance and synaptic plasticity (Fields \& StevensGraham, 2002). Neuroglial cells contribute in a number of ways to the regulation of immune responses in the CNS. Astrocytes, for example, play an important role in the detoxification of excess excitatory amino acids (Nedergaard, Takano, \& Hansen, 2002), maintenance of the integrity of the blood-brain barrier (Prat et al., 2001), and production of neurotrophic factors (Bauer, Rauschka, \& Lassmann, 2001). In normal homeostatic conditions, astrocytes facilitate neuronal survival by producing growth factors and mediating uptake/removal of excitotoxic neurotransmitters, such as glutamate, from the synaptic microenvironment (Nedergaard, Takano, \& Hansen, 2002).

However, during astroglial activation secondary to injury or in response to neuronal dysfunction, astrocytes can produce several factors that may modulate inflammatory responses; they secrete proinflammatory cytokines, chemokines, and metalloproteinases that can magnify immune reactions within the CNS (Bauer, Rauschka, \& Lassmann, 2001; Rosenberg, 2002). Similarly, microglial activation is an important factor in the neuroglial responses to injury or dysfunction. Microglia are involved in synaptic stripping, cortical plasticity, and immune surveillance (Aloisi, 2001). Changes in astroglia and microglia can therefore produce marked neuronal and synaptic changes that are likely to contribute to CNS dysfunction or modify CNS homeostasis during disease processes. Neuronal dysfunction and abnormalities in cortical organization such as those seen in autism may also be responsible for pathophysiological responses that may lead to neuroglial activation, reactions that may subsequently increase the magnitude of neuronal dysfunction.

\section{Neuroglia responses in autism}

The role of neuroglia in autism has been ignored in the past several years and previous neuropathological studies did not show evidence of astrogliosis or microglial reactions (Kemper \& Bauman, 1998). Evidence of neuroglial activation and a role for neuroimmune responses mediated by innate immunity in the neuropathology of autism, recently has been demonstrated by our laboratory (Vargas et al., 2005). Based on neuropathological analysis of postmortem brain tissues from 11 autistic patients (age range 5-44 years), we have demonstrated the presence of an active and ongoing neuroinflammatory process in the cerebral cortex and white matter, and notably in the cerebellum. Immunocytochemical studies of brain tissues from these 11 autistic patients showed marked activation of microglia and astroglia as compared with controls. The neuroglial activation was particularly prominent in the granular cell layer and white matter of the cerebellum. An assessment of the magnitude of astrogliosis using immunocytochemistry for glial fibrillary acidic protein (GFAP) in the midfrontal (MFG) and anterior cingulate gyrus (ACG) and cerebellum (CBL) of the autistic brains revealed increased astroglial reactions characterized by an increase in the volume of perikarya and glial processes. In the brains of autistic patients, GFAP immunostaining of the cerebellum showed a marked reactivity of the Bergmann's astroglia in areas of neuronal loss within the Purkinje cell layer, as well as a marked astroglial reaction in the granular cell layer and cerebellar white matter. In the MFG and ACG, astroglial reactions were prominent in the subcortical white matter, and in some cases panlaminar astrogliosis was observed. Quantitative assessment of astroglial immunoreactivity by fractional area methods showed a significant increase in GFAP immunoreactivity in the GCL $(P=0.000)$ and white matter $(P=0.007)$ compartments of the cerebellum. Further analysis by western blotting of GFAP expression in protein homogenates obtained from a subset of autistic $(n=7)$ and control patients $(n=7)$ from whom fresh-frozen brain tissue had been obtained, showed a significantly increased expression of GFAP in the cerebellum $(P=0.001)$, MFG $(P=0.001)$ and ACG $(P=0.038)$ of autistic patients, as compared to controls, findings that demonstrate the presence of a marked astroglial reaction in autism.

The pattern of microglial activation in autistic brains was further characterized by immunocytochemical staining for MHC class II markers (HLA-DR). Marked microglial activation was observed in the cerebellum, cortical regions and white matter of autistic patients. The most prominent microglial reaction was observed in the cerebellum, where the immunoreactivity for HLA-DR showed a significantly higher fractional area of immunoreactivity in both the GCL $(P<0.0001)$ and cerebellar white matter $(P<0.0001)$ of autistic subjects than in controls 


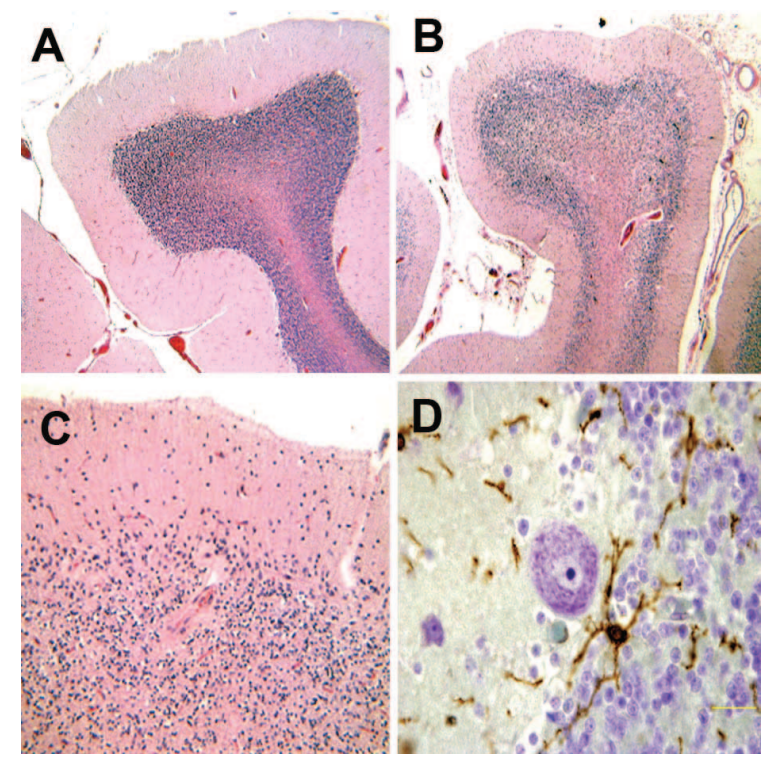

Figure 1. Neuropathology of cerebellum in autism. (A) Normal appearance of the cerebellum in a control patient; (B-C) atrophic folia and marked loss of Purkinje and granular cells in the cerebellum of an autistic patient (H\&E stain); (D) microglia activation seen with anti-MHC class II immunostaining (from Vargas et al., 2005).

(Figure 1). At present, it is still unclear what the role of neuroglial responses in autism is or how these responses are involved in pathogenic mechanisms. The microglial and astroglial activation in the CNS may then have a dichotomous role in the inflammatory responses of the brain: as a direct effector of injury and on the other hand as neuroprotectant (Nguyen, Julien, \& Rivest, 2002). It is unclear how and when microglia and astroglia become activated in the brain of autistic patients. Neuroglial activation in autism may be part of both primary (intrinsic) responses that result from disturbances of neuroglial function or neuronal-neuroglial interactions during brain development and secondary (extrinsic) effects, resulting from unknown factors that disturb prenatal or postnatal CNS development. It is possible that the presence of activated microglia in the brain in autism may reflect abnormal persistence of fetal patterns of development in response to genetic or environmental (e.g., intrauterine, maternal) factors. Our findings may indicate that at some point during cortical and neuronal organization, unknown factors influence both neuronal and neuroglial cell populations, disturbing neurodevelopment and producing the neurocytoarchitectural changes seen in autism as well as inducing CNS dysfunction that results in neuroinflammation. Another potential explanation is that extrinsic etiological factors (e.g., non-genetic, neurotoxic or environmental) involved in the pathogenesis of autism may produce neuronal and cortical abnormalities, to which neuroglial reactions are only secondary responses.

\section{Cytokine profile in the brain of autistic patients}

Cytokines and chemokines play important roles as mediators of inflammatory reactions in the CNS and in processes of neuronal-neuroglial interactions that modulate the neuroimmune system. Cytokines may contribute to neuroinflammation as mediators of pro-inflammatory or anti-inflammatory responses within the CNS. Our laboratory has focused on studies to characterize the profiles of cytokines and chemokines in autistic brains by assessing the relative expression of these proteins in tissue homogenates from MFG, ACG, and CBL of autistic $(n=7)$ and control $(n=7)$ patients by using cytokine protein array methodology (Huang, 2004). A statistical analysis of the relative expression of cytokines in autistic and control tissues showed a consistent and significantly higher level of subsets of cytokines in the brains of autistic patients: the anti-inflammatory cytokine transforming growth factor $\beta 1$ (TGF- $\beta 1$ ) was increased in the MFG $(P=0.026)$, ACG $(P=0.011)$ and CBL $(P=0.035)$ and the proinflammatory chemokines macrophage chemoattractant protein-1 (MCP-1) and thymus and activation-regulated chemokine (TARC), were increased in the ACG $(P=0.026$ and 0.035, respectively) and CBL $(P=0.026$ and 0.035 , respectively). Interestingly, a larger spectrum of increases in pro-inflammatory and modulatory cytokines was seen in the ACG, an important cortical structure in autism, where there was a significant increase in pro-inflamamtory cytokines such as interleukin-6 (IL-6), interleukin-10 (IL-10), macrophage chemoattractant protein-3 (MCP-3), eotaxin, eotaxin 2, macrophage-derived chemokine (MDC), chemokine- $\beta 8 \quad(\mathrm{Ck} \beta 8.1)$, neutrophil activating peptide-2 (NAP-2), monokine induced by interferon- $\gamma$ (MIG) and B-lymphocyte chemoattractant (BLC) (Figure 2).

The presence of MCP-1 is of particular interest, since it facilitates the infiltration and accumulation of monocytes and macrophages in inflammatory CNS disease (Mahad \& Ransohoff, 2003). Chemoattractant protein-1 is produced by activated and reactive astrocytes, a finding that demonstrate the effector role of these cells in the disease process in autism. The increase in MCP-1 expression has relevance to the pathogenesis of autism as we believe its elevation in the brain is linked to pathways of microglial activation and perhaps to the recruitment of monocytes/macrophages to areas of neuronalcortical abnormalities. Our observations resemble findings in other neurological disorders in 


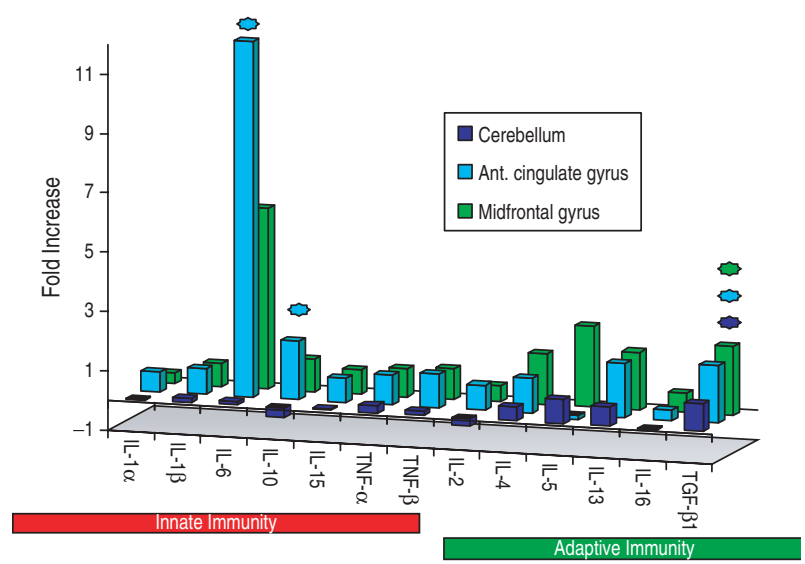

Figure 2. Pattern of increase of cytokines in brain regions of autistic patients (from Vargas et al., 2005).

which elevation of MCP-1 is associated with the pathogenesis of neuroinflammation and neuronal injury such as HIV dementia (Kelder et al., 1998), ALS (Henkel et al., 2004), and multiple sclerosis (Mahad \& Ransohoff, 2003). It remains unclear whether MCP-1 plays a more pleotrophic role in the CNS or whether its presence is only associated with inflammatory conditions. The presence of increased TGF- $\beta 1$ in the cortex and cerebellum of autistic brains may have important implications for the neurobiology of autism. Transforming growth factor $\beta 1$ is a key anti-inflammatory cytokine and is involved in tissue remodeling following injury. It can suppress specific immune responses by inhibiting T-cell proliferation and maturation and downregulates MHC class II expression (Letterio \& Roberts, 1998). Importantly, cells undergoing cell death have been shown to secrete TGF- $\beta 1$, possibly to reduce local inflammation and prevent degeneration of additional surrounding cells (Chen et al., 2001). Transforming growth factor $\beta 1$ is produced mostly by reactive astrocytes and neurons. The elevation of TGF- $\beta 1$ suggests that the elevation of this cytokine in autism may reflect an attempt to modulate neuroinflammation or remodel and repair injured tissue. A remarkable profile of cytokine up-regulation was observed in the ACG, a region in which several cytokines, chemokines, and growth factors were markedly elevated when compared to controls. Pro-inflammatory cytokines (e.g., IL-6) and anti-inflammatory cytokines (e.g., IL-10) as well as subsets of chemokines were markedly elevated in the ACG, an important cortical region involved in dysfunctional brain activity in autism. These findings support the conclusion that an active, ongoing immunological process was present in multiple areas of the brain but at different levels of expression in each area.

\section{Cerebrospinal fluid and neuroinflammation in autism}

Despite these indications of multiple peripheral immune system abnormalities, there has been no consistent demonstration of inflammatory changes in cerebrospinal fluid (CSF) studies, and there has been no evidence of inflammation as indicated by standard cell counts, protein electrophoresis, protein concentration, increase of IgG index, or presence of oligoclonal bands (Zimmerman et al., 2005). We have recently studied the profile of cytokines and chemokines in the CSF of autistic patients as an approach to evaluate proteins involved in innate and adaptive immune pathways (Vargas et al., 2005). Cytokine protein arrays were used to compare the cytokine profiles of CSF from six autistic patients to that of CSF from a pool of donors without CNS pathology or inflammatory disorders (e.g., pseudotumor cerebri or headaches). We observed a marked increase in subsets of cytokines and chemokines involved in innate immune responses. As we had observed in brain tissues, CSF from autistic patients showed a significant increase in MCP-1 (12-fold increase) when compared to controls. Other pro-inflammatory such as IL-6, IFN- $\gamma$, IL-8, macrophage inflammatory protein- $1 \beta$ (MIP1 $\beta)$, NAP-2, interferon- $\gamma$ inducing protein-10 (IP-10) and angiogenin were all significantly increased when compared to control CSF (Figure 3). These cytokines play important roles in immune-mediated processes and their presence in the CSF in autistic patients may reflect an ongoing stage of inflammatory reactions likely associated with neuroglial activation and/or neuronal injury. Reasons for the relatively greater increases in these cytokines in CSF compared to brain are unknown. The differences we observed in cytokines in CSF compared to brain could result from other sources of production, such the leptomeninges or choroid plexus or might represent a persistent elevation of cytokines as result of a stage of neurodevelopmental arrest as some of the cytokines are normally elevated during phases of neurodevelopment. Since the CSF is easily accessible for clinical studies, CSF cytokine profiling may be useful in the future to diagnose, characterize and follow the clinical course of autistic disorders.

\section{Clinical and therapeutic implications of neuroinflammation in autism}

Evaluation of neuroinflammation in autistic patients and its use in clinical assessment raises an important challenge. The classical techniques in the evaluation of CSF in autistic patients have failed in providing information about the presence of 

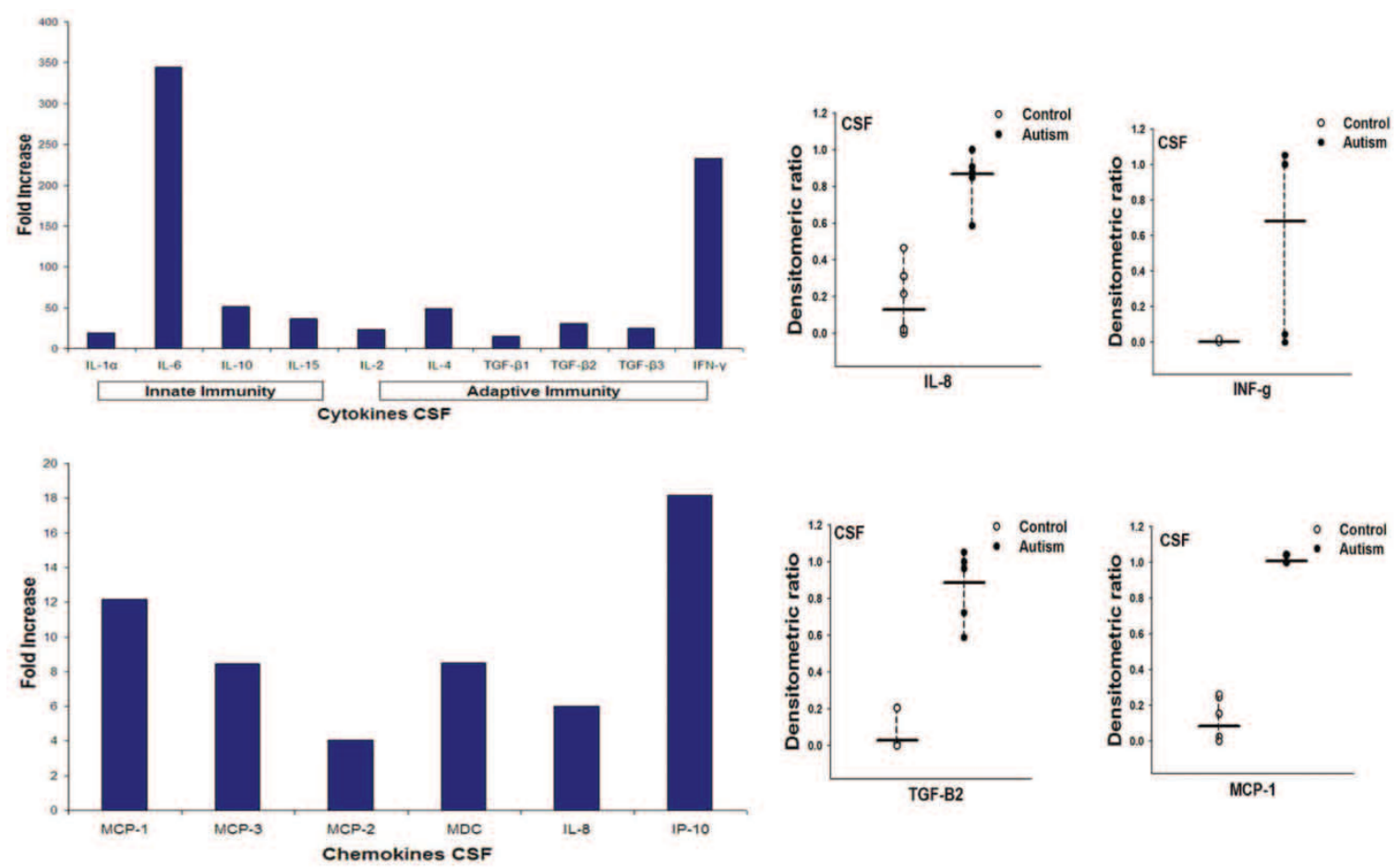

Figure 3. Pattern of increase in cytokines and chemokines in the CSF of autistic patients (from Vargas et al., 2005).

inflammatory changes as there is not evidence of either pleocytosis, cellular reactions or humoral responses such as increase in immunoglobulin index or oligoclonal bands. Recent studies using detection of products of macrophage and immune reactions such as neopterin, quinolinic acid or biopterin were shown to be unhelpful in the detection of neuroinflammation in autistic patients (Zimmerman et al., 2005). Our observations that subsets of cytokines and chemokines such as IL-6, IFN- $\gamma$ and MCP-1 are elevated in patients with autism suggest that assessment of cytokine profiles are a potential approach to identify and evaluate the magnitude of inflammatory responses in these patients. It remains unknown whether these profiles correlate with the clinical spectrum of autism and further studies are required to understand the role of these cytokines and chemokines in the disease process. Another approach that may become practical in the future is the use of novel neuroimaging techniques such as brain imaging using PK11195, a ligand to the benzodiazepine receptor as a marker of microglial activation in vivo to determine the magnitude and extension of neuroglial reactions (Versijpt et al., 2003).

Another issue that is extremely important is to determine whether neuro-inflamamtion and neuroglial activation may be a target for treatment in autism. This issue requires a more detailed evaluation as the precise role of neuroinflammation in the pathogenesis and natural history of autism is still uncertain. Studies in animal models and other neurological disorders suggest that microglial activation and neuroinflammation may play a role in processes of injury as there is increased oxidative stress and tissue injury, however, there is also recent evidence that neuroinflammation may be associated with repair processes and regeneration (Neuhaus, Archelos, \& Hartung, 2003). Further studies are required in autism to help in the clarification of these issues. So, at this moment, we consider premature the use of any immunomodulatory intervention to modify the neuroglial activation and neuroinflammation. Furthermore, current treatment approaches to modify neuroimmune responses are very nonspecific and may bring more potential problems than benefits. The use of steroids or other immunotherapies such as immunoglobulin infusion and use of cytotoxic drugs may bring potential risks as these medications act mostly in cellular and humoral responses that are part of the adaptive immune system rather than neuroglial activation or innate immune responses.

\section{Future directions}

Several important questions regarding the role of neuroimmunity in autism remain unanswered, including: (1) Whether the neuroglial and neuroimmune responses associated with autism are part of the primary reactions that contribute to CNS dysfunction in this disorder or are epiphenomena 


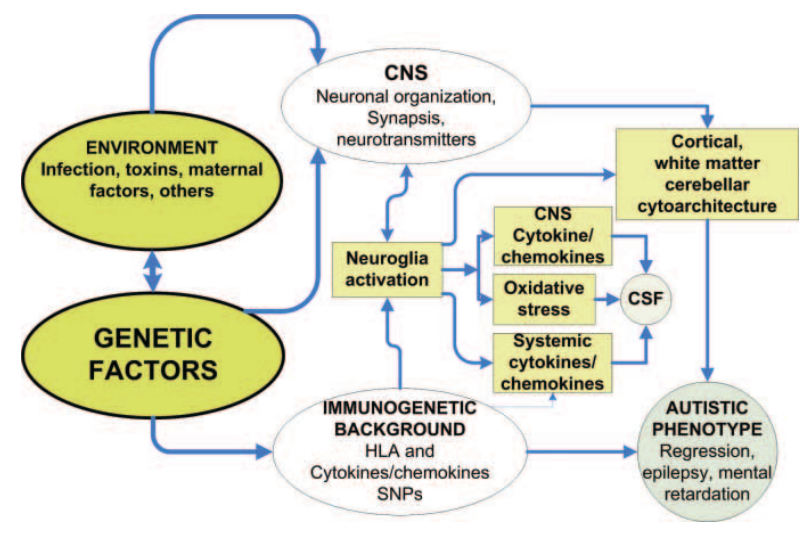

Figure 4. Hypothetical interactions of environmental and genetic factors that influence neuroglia activation, CNS organization and the presence of autism.

resulting from reactions to CNS dysfunction; (2) the nature of the relationship of cytokines and chemokines to immune and neurobiological processes in the brain of autistic patients; (3) whether the cerebellar pathology in autism is primarily the result of neuroimmune processes or primary abnormalities in neuronal function; (4) how analysis of CSF may help us identify markers of immune reactions within the CNS; and (5) whether the immunogenetic background of the host influences the development of neuroimmune reactions or determines patterns of susceptibility to autism.

\section{Conclusions}

Autism is a complex neurobehavioral disorder of early life onset influenced by the interaction of different risk factors. We hypothesize that environmental factors (e.g., neurotoxins, infections, maternal infections) in presence of genetic susceptibility and the immunogenetic background of the host influence the development of abnormalities in cortical organization and neuronal circuitry and neuroinflammatory changes responsible for the generation of the autistic symptoms (Figure 4). Our neuroimmunopathological studies strongly suggests that innate rather than adaptive neuroimmune responses are part of the immunopathogenic mechanisms associated with autism, but we cannot exclude the possibility that specific immune reactions, cellular or humoral, may occur at early stages of the disease, during prenatal or postnatal stages of brain development. The roles of neuroglial activation and neuroinflammation in the pathogenesis of autism are still uncertain but could be critical in maintaining, if not also in initiating, some of the CNS abnormalities present in this neurodevelopmental disorder. Neuroglial and neuroinflammatory responses likely have polygenic and environmental bases and may have important clinical and therapeutic implications in autism.

\section{Acknowledgements}

The authors are grateful for the support received from the Cure Autism Now Foundation (CAN), by Dr. Barry and Mrs. Renee Gordon, Dr. Jane Pickett and the Autism Tissue Program. Dr. Pardo is supported by a grant from NIH-NIDA (K08-DA 16160-01a1).

\section{References}

Aloisi, F. (2001). Immune function of microglia. Glia, 36, 165-179.

Aman, M. G., Novotny, S., Samango-Sprouse, C., Lecavalier, L., Leonard, E., Gadow, K. D., et al. (2004). Outcome measures for clinical drug trials in autism. CNS Spectrums, 9, 36-47.

Ashwood, P., \& Van de Water, J. (2004). Is autism an autoimmune disease? Autoimmunity Reviews, 3, 557-562.

Bailey, A., Luthert, P., Dean, A., Harding, B., Janota, I., Montgomery, M., et al. (1998). A clinicopathological study of autism. Brain, 121(Pt 5), 889-905.

Barbaresi, W. J., Katusic, S. K., Colligan, R. C., Weaver, A. L., \& Jacobsen, S. J. (2005). The incidence of autism in Olmsted County, Minnesota, 1976-1997: Results from a populationbased study. Archives of Pediatric \& Adolescent Medicine, 159, 37-44.

Barnea-Goraly, N., Kwon, H., Menon, V., Eliez, S., Lotspeich, L., \& Reiss, A. L. (2004). White matter structure in autism: Preliminary evidence from diffusion tensor imaging. Biological Psychiatry, 55, 323-326.

Bauer, J., Rauschka, H., \& Lassmann, H. (2001). Inflammation in the nervous system: The human perspective. Glia, 36, 235-243.

Becker, K. G., Freidlin, B., \& Simon, R. M. (2003). Comparative genomics of autism, Tourette syndrome and autoimmune/ inflammatory disorders. www.grc.nia.nih.gov/branches/rrb/dna/ pubs/cgoatad.pdf.

Carbone, K. M., Rubin, S. A., \& Pletnikov, M. (2002). Borna disease virus (BDV)-induced model of autism: Application to vaccine safety test design. Molecular Psychiatry, 7 (Suppl. 2), S36-S37.

Casanova, M. F., Buxhoeveden, D. P., Switala, A. E., \& Roy, E. (2002). Minicolumnar pathology in autism. Neurology, 58, 428-432.

Chen, W., Frank, M. E., Jin, W., \& Wahl, S. M. (2001). TGFbeta released by apoptotic $\mathrm{T}$ cells contributes to an immunosuppressive milieu. Immunity, 14, 715-725.

Chess, S., Fernandez, P., \& Korn, S. (1978). Behavioral consequences of congenital rubella. Fournal of Pediatrics, 93, 699-703.

Cohen, D., Pichard, N., Tordjman, S., Baumann, C., Burglen, L., Excoiffier, E., et al. (2005). Specific genetic disorders and autism: Clinical contribution towards their identification. Fournal of Autism \& Developmental Disorders, 35, 103-116.

Comi, A. M., Zimmerman, A. W., Frye, V. H., Law, P. A., \& Peeden, J. N. (1999). Familial clustering of autoimmune disorders and evaluation of medical risk factors in autism. fournal of Child Neurology, 14, 388-394.

Connolly, A. M., Chez, M. G., Pestronk, A., Arnold, S. T., Mehta, S., \& Deuel, R. K. (1999). Serum autoantibodies to 
brain in Landau-Kleffner variant, autism, and other neurologic disorders. Fournal of Pediatrics, 134, 607-613.

Courchesne, E., \& Pierce, K. (2005). Brain overgrowth in autism during a critical time in development: Implications for frontal pyramidal neuron and interneuron development and connectivity. International fournal of Developmental Neuroscience, 23, 153-170.

Courchesne, E., Redcay, E., \& Kennedy, D. P. (2004). The autistic brain: Birth through adulthood. Current Opinions in Neurology, 17, 489-496.

Croen, L. A., Grether, J. K., Yoshida, C. K., Odouli, R., \& Van de, W. J. (2005). Maternal autoimmune diseases, asthma and allergies, and childhood autism spectrum disorders: A casecontrol study. Archives in Pediatric \& Adolescent Medicine, 159, 151-157.

Dalton, P., Deacon, R., Blamire, A., Pike, M., McKinlay, I., Stein, J., et al. (2003). Maternal neuronal antibodies associated with autism and a language disorder. Annal of Neurology, 53, 533-537.

Daniels, W. W., Warren, R. P., Odell, J. D., Maciulis, A., Burger, R. A., Warren, W. L., et al. (1995). Increased frequency of the extended or ancestral haplotype B44-SC30DR4 in autism. Neuropsychobiology, 32, 120-123.

DeLong, G. R., Bean, S. C., \& Brown, F. R. III. (1981). Acquired reversible autistic syndrome in acute encephalopathic illness in children. Archives in Neurology, 38, 191-194.

Dong, Y., \& Benveniste, E. N. (2001). Immune function of astrocytes. Glia, 36, 180-190.

Fields, R. D., \& Stevens-Graham, B. (2002). New insights into neuron-glia communication. Science, 298, 556-562.

Folstein, S. E., \& Rosen-Sheidley, B. (2001). Genetics of autism: Complex aetiology for a heterogeneous disorder. Nature Reviews Genetics, 2, 943-955.

Fombonne, E. (2003). Epidemiological surveys of autism and other pervasive developmental disorders: An update. Fournal of Autism \& Developmental Disorders, 33, 365-382.

Guerin, P., Lyon, G., Barthelemy, C., Sostak, E., Chevrollier, V., Garreau, B., et al. (1996). Neuropathological study of a case of autistic syndrome with severe mental retardation. Developmental Medicine E Child Neurology, 38, 203-211.

Gupta, S., Aggarwal, S., Rashanravan, B., \& Lee, T. (1998). Th1- and Th2-like cytokines in CD4+ and CD8+ T cells in autism. fournal of Neuroimmunology, 85, 106-109.

Halsey, N. A., \& Hyman, S. L. (2001). Measles-mumps-rubella vaccine and autistic spectrum disorder: Report from the New Challenges in Childhood Immunizations Conference convened in Oak Brook, Illinois, June 12-13, 2000. Pediatrics, 107, E84.

Hashimoto, T., Tayama, M., Murakawa, K., Yoshimoto, T., Miyazaki, M., Harada, M., et al. (1995). Development of the brainstem and cerebellum in autistic patients. Fournal of Autism E Developmental Disorders, 25, 1-18.

Henkel, J. S., Engelhardt, J. I., Siklos, L., Simpson, E. P., Kim, S. H., Pan, T., et al. (2004). Presence of dendritic cells, MCP-1, and activated microglia/macrophages in amyotrophic lateral sclerosis spinal cord tissue. Annals of Neurology, 55, 221-235.

Herbert, M. R., Ziegler, D. A., Deutsch, C. K., O’Brien, L. M., Lange, N., Bakardjiev, A., et al. (2003). Dissociations of cerebral cortex, subcortical and cerebral white matter volumes in autistic boys. Brain, 126, 1182-1192.

Herbert, M. R., Ziegler, D. A., Makris, N., Filipek, P. A., Kemper, T. L., Normandin, J. J., et al. (2004). Localization of white matter volume increase in autism and developmental language disorder. Annals of Neurology, 55, 530-540.

Huang, R. P. (2004). Cytokine protein arrays. Methods in Molecular Biology, 278, 215-232.

Jyonouchi, H., Sun, S., \& Le, H. (2001). Pro-inflammatory and regulatory cytokine production associated with innate and adaptive immune responses in children with autism spectrum disorders and developmental regression. fournal of Neuroimmunology, 120, 170-179.

Kaufmann, W. E., Cooper, K. L., Mostofsky, S. H., Capone, G. T., Kates, W. R., Newschaffer, C. J., et al. (2003). Specificity of cerebellar vermian abnormalities in autism: A quantitative magnetic resonance imaging study. Fournal of Child Neurology, 18, 463-470.

Kawashima, H., Mori, T., Kashiwagi, Y., Takekuma, K., Hoshika, A., \& Wakefield, A. (2000). Detection and sequencing of measles virus from peripheral mononuclear cells from patients with inflammatory bowel disease and autism. Digestive Diseases E Science, 45, 723-729.

Kelder, W., McArthur, J. C., Nance-Sproson, T., McClernon, D., \& Griffin, D. E. (1998). Beta-chemokines MCP-1 and RANTES are selectively increased in cerebrospinal fluid of patients with human immunodeficiency virus-associated dementia. Annals of Neurology, 44, 831-835.

Kemper, T. L., \& Bauman, M. (1998). Neuropathology of infantile autism. Fournal of Neuropathology \& Experimental Neurology, 57, 645-652.

Korvatska, E., Van de, W. J., Anders, T. F., \& Gershwin, M. E. (2002). Genetic and immunologic considerations in autism. Neurobiology of Disease, 9, 107-125.

Larsson, H. J., Eaton, W. W., Madsen, K. M., Vestergaard, M., Olesen, A. V., Agerbo, E., et al. (2005). Risk factors for autism: Perinatal factors, parental psychiatric history, and socioeconomic status. American fournal of Epidemiology, 161, 916-925.

Lee, L.-C., Zachary, A. A., Leffell, M. S., Newschaffer, C. J., Matteson, K. J., Tyler, J. D., et al. (2004). Increased incidence of maternal HLA-DR4 in single-birth, but not multiplex families with autism. International Meeting for Autism Research, Sacramento, CA. May 2004 (abstr).

Letterio, J. J., \& Roberts, A. B. (1998). Regulation of immune responses by TGF-beta. Annual Review of Immunology, 16, 137-161.

Levin, L., Ban, Y., Concepcion, E., Davies, T. F., Greenberg, D. A., \& Tomer, Y. (2004). Analysis of HLA genes in families with autoimmune diabetes and thyroiditis. Human Immunology, 65, 640-647.

Lord, C., Cook, E. H., Leventhal, B. L., \& Amaral, D. G. (2000). Autism spectrum disorders. Neuron, 28, 355-363.

Lord, C., Shulman, C., \& DiLavore, P. (2004). Regression and word loss in autistic spectrum disorders. Fournal of Child Psychology \& Psychiatry, 45, 936-955.

Mahad, D. J., \& Ransohoff, R. M. (2003). The role of MCP-1 (CCL2) and CCR2 in multiple sclerosis and experimental autoimmune encephalomyelitis (EAE). Seminars in Immunology, $15,23-32$.

Micali, N., Chakrabarti, S., \& Fombonne, E. (2004). The broad autism phenotype: Findings from an epidemiological survey. Autism, 8, 21-37.

Money, J., Bobrow, N. A., \& Clarke, F. C. (1971). Autism and autoimmune disease: A family study. Fournal of Autism \& Child Schizophrenia, 1, 146-160.

Nedergaard, M., Takano, T., \& Hansen, A. J. (2002). Beyond the role of glutamate as a neurotransmitter. Nature Reviews Neuroscience, 3, 748-755.

Neuhaus, O., Archelos, J. J., \& Hartung, H. P. (2003). Immunomodulation in multiple sclerosis: From immunosuppression to neuroprotection. Trends in Pharmacological Science, 24, 131-138.

Newschaffer, C. J., Fallin, D., \& Lee, N. L. (2002). Heritable and non-heritable risk factors for autism spectrum disorders. Epidemiology Reviews, 24, 137-153.

Nguyen, M. D., Julien, J. P., \& Rivest, S. (2002). Innate immunity: The missing link in neuroprotection and neurodegeneration? Nature Reviews Neuroscience, 3, 216-227. 
Odell, D., Maciulis, A., Cutler, A., Warren, L., McMahon, W. M., Coon, H., et al. (2005). Confirmation of the association of the C4B null allelle in autism. Human Immunology, 66, 140-145.

Patterson, P. H. (2002). Maternal infection: Window on neuroimmune interactions in fetal brain development and mental illness. Current Opinions in Neurobiology, 12, 115-118.

Prat, A., Biernacki, K., Wosik, K., \& Antel, J. P. (2001). Glial cell influence on the human blood-brain barrier. Glia, 36, 145-155.

Rapin, I. (1997). Autism. New England Fournal of Medicine, 337, 97-104.

Rapin, I., \& Katzman, R. (1998). Neurobiology of autism. Annals of Neurology, 43, 7-14.

Rose, N. R., \& Bona, C. (1993). Defining criteria for autoimmune diseases (Witebsky's postulates revisited). Immunology Today, 14, 426-430.

Rosenberg, G. A. (2002). Matrix metalloproteinases in neuroinflammation. Glia, 39, 279-291.

Shi, L., Fatemi, S. H., Sidwell, R. W., \& Patterson, P. H. (2003). Maternal influenza infection causes marked behavioral and pharmacological changes in the offspring. Fournal of Neuroscience, 23, 297-302.

Silva, S. C., Correia, C., Fesel, C., Barreto, M., Coutinho, A. M., Marques, C., et al. (2004). Autoantibody repertoires to brain tissue in autism nuclear families. Fournal of Neuroimmunology, 152, 176-182.

Singh, V. K., Lin, S. X., Newell, E., \& Nelson, C. (2002). Abnormal measles-mumps-rubella antibodies and CNS autoimmunity in children with autism. Fournal of Biomedical Science, 9, 359-364.

Singh, V. K., Lin, S. X., \& Yang, V. C. (1998). Serological association of measles virus and human herpesvirus-6 with brain auto-antibodies in autism. Clinical Immunology $\mathcal{E}$ Immunopathology, 89, 105-108.

Singh, V. K., Warren, R., Averett, R., \& Ghaziuddin, M. (1997). Circulating autoantibodies to neuronal and glial filament proteins in autism. Pediatric Neurology, 17, 88-90.

Stubbs, E. G., \& Crawford, M. L. (1977). Depressed lymphocyte responsiveness in autistic children. Fournal of Autism $\mathcal{E}$ Child Schizophrenia, 7, 49-55.

Sweeten, T. L., Bowyer, S. L., Posey, D. J., Halberstadt, G. M., \& McDougle, C. J. (2003). Increased prevalence of familial autoimmunity in probands with pervasive developmental disorders. Pediatrics, 112, e420.

Todd, R. D., Hickok, J. M., Anderson, G. M., \& Cohen, D. J. (1988). Antibrain antibodies in infantile autism. Biological Psychiatry, 23, 644-647.

Torres, A. R., Maciulis, A., Stubbs, E. G., Cutler, A., \& Odell, D. (2002). The transmission disequilibrium test suggests that HLA-DR4 and DR13 are linked to autism spectrum disorder. Human Immunology, 63, 311-316.
Tuchman, R., \& Rapin, I. (2002). Epilepsy in autism. Lancet Neurology, 1, 352-358.

van Gent, T., Heijnen, C. J., \& Treffers, P. D. (1997). Autism and the immune system. Fournal of Child Psychology \& Psychiatry, 38, 337-349.

Vargas, D. L., Nascimbene, C., Krishnan, C., Zimmerman, A. W., \& Pardo, C. A. (2005). Neuroglial activation and neuroinflammation in the brain of patients with autism. Annals of Neurology, 57, 67-81.

Versijpt, J. J., Dumont, F., Van Laere, K. J., Decoo, D., Santens, P., Audenaert, K., et al. (2003). Assessment of neuroinflammation and microglial activation in Alzheimer's disease with radiolabelled PK11195 and single photon emission computed tomography. A pilot study. European fournal of Neurology, 50, 39-47.

Vojdani, A., Campbell, A. W., Anyanwu, E., Kashanian, A., Bock, K., \& Vojdani, E. (2002). Antibodies to neuron-specific antigens in children with autism: Possible cross-reaction with encephalitogenic proteins from milk, Chlamydia pneumoniae and Streptococcus group A. Fournal of Neuroimmunology, 129, 168-177.

Warren, R. P., Cole, P., Odell, J. D., Pingree, C. B., Warren, W. L., White, E., et al. (1990). Detection of maternal antibodies in infantile autism. Fournal of the American Academy of Child $\mathbb{E}$ Adolescent Psychiatry, 29, 873-877.

Warren, R. P., Margaretten, N. C., Pace, N. C., \& Foster, A. (1986). Immune abnormalities in patients with autism. fournal of Autism \& Developmental Disorders, 16, 189-197.

Wing, L., \& Potter, D. (2002). The epidemiology of autistic spectrum disorders: Is the prevalence rising? Mental Retardation \& Developmental Disabilities Research Reviews, 8, 151-161.

Yamashita, Y., Fujimoto, C., Nakajima, E., Isagai, T., \& Matsuishi, T. (2003). Possible association between congenital cytomegalovirus infection and autistic disorder. Fournal of Autism E Developmental Disorders, 33, 455-459.

Yeargin-Allsopp, M., Rice, C., Karapurkar, T., Doernberg, N., Boyle, C., \& Murphy, C. (2003). Prevalence of autism in a US metropolitan area. Fournal of the American Medical Association, 289, 49-55.

Zimmerman, A. W., Jyonouchi, H., Comi, A. M., Connors, S. L., Milstien, S., Varsou, A., et al. (2005). Cerebrospinal fluid and serum markers of inflammation in autism. Pediatric Neurology, 33, 195-201.

Zimmerman, A. W., Tyler, J. D., \& Matteson, K. J. (2001). Increased incidence of HLA-B60 and maternal DR4 in autism. Annals of Neurology, 50, S122-S123.

Zimmerman, A. W. (2005). The immune system. In M. Bauman \& T. L. Kemper (Eds.), The neurobiology of autism (pp. 371-386). Baltimore: The Johns Hopkins University Press. 